\title{
Provision of cross-domain knowledge in mechanical joining using ontologies
}

\author{
Christoph Zirngib| ${ }^{1}$ - Patricia Kügler ${ }^{1}$ Julian Popp ${ }^{2} \cdot$ Christian R. Bielak $^{3} \cdot$ Mathias Bobbert $^{3} \cdot$ Dietmar Drummer $^{2}$. \\ Gerson Meschut ${ }^{3}$. Sandro Wartzack ${ }^{1} \cdot$ Benjamin Schleich ${ }^{1}$
}

Received: 15 October 2021 / Accepted: 7 February 2022 / Published online: 24 February 2022

(c) The Author(s) 2022

\begin{abstract}
Since the application of mechanical joining methods, such as clinching or riveting, offers a robust solution for the generation of advanced multi-material connections, the use in the field of lightweight designs (e.g. automotive industry) is steadily increasing. Therefore, not only the design of an individual joint is required but also the dimensioning of the entire joining connection is crucial. However, in comparison to thermal joining techniques, such as spot welding, the evaluation of the joints' resistance against defined requirements (e.g. types of load, minimal amount of load cycles) mainly relies on the consideration of expert knowledge, a few design principles and a small amount of experimental data. Since this generally implies the involvement of several domains, such as the material characterization or the part design, a tremendous amount of data and knowledge is separately generated for a certain dimensioning process. Nevertheless, the lack of formalization and standardization in representing the gained knowledge leads to a difficult and inconsistent reuse, sharing or searching of already existing information. Thus, this contribution presents a specific ontology for the provision of cross-domain knowledge about mechanical joining processes and highlights two potential use cases of this ontology in the design of clinched and pin joints.
\end{abstract}

Keywords Mechanical joining process $\cdot$ Pin-joining $\cdot$ Clinching $\cdot$ Cross-domain knowledge $\cdot$ Ontology

\section{Introduction}

The benefits of mechanical joining processes, such as their high cost- and time-efficiency as well as their possibility to join dissimilar materials with or without the use of auxiliary joining elements, lead to an increased use of such processes, particularly in the context of lightweight design. Additionally, their environmentally friendliness and the lower energy consumption enable to achieve emission targets and regulatory requirements. However, besides the selection of a suitable process, the dimensioning and design of the individual

Christoph Zirngibl

zirngibl@mfk.fau.de

1 Engineering Design (KTmfk), Friedrich-AlexanderUniversität Erlangen-Nürnberg (FAU), Martensstraße 9, 91058 Erlangen, Germany

2 Institute of Polymer Technology, Friedrich-Alexander-Uni versität Erlangen-Nürnberg (FAU), Am Weichselgarten 9, 91058 Erlangen, Germany

3 Laboratory for Material and Joining Technology, University Paderborn, Pohlweg 47-49, 33098 Paderborn, Germany joining connection regarding external influences, such as tensile and shear loading, or construction limits are the most crucial steps. Therefore, to ensure a high reliability of the joint, often several domains, such as material characterization, numerical simulation and part design have to be taken into consideration involving a wide range of specific and sequential analysis steps. Since a lack of formalization and standardization within the generated and already existing knowledge can lead to misinterpretations and limitations in searching for the required information, a consistent and efficient transfer of knowledge between the domains is hardly achievable. Additionally, the inadequate sharing of results and the absence of consistency in the terminology prevent the comparative evaluation and reuse of already available knowledge from different sources. Thus, based on the limited access to knowledge databases, it is often necessary to repeat resource-intensive and time-consuming dimensioning and design steps for the identification of best-fitting joint configurations. However, a method for the explicit specification of cross-domain and shared mechanical joining understanding is not available yet. As a solution to this problem, the setup of an explicit ontology showed already 
high potentials in several application fields (e. g. in the domain of tribology) by providing formal defined concepts. Therefore, the formalization of data creates new opportunities for intensifying and supporting the sharing and reuse of knowledge in the domain of mechanical joining processes. Thus, the resulting machine-processability provides a basis for analyzing and querying design, experimental and simulation setups and data.

\section{Related work}

Applying ontologies for the purpose of reuse, sharing and improving interoperability of knowledge sources is not new within mechanical domains. Saha et al. [1] introduced a core domain ontology for joining processes focusing on welding standards. The ontology is intended to overcome the terminological inconsistencies within and across those standards and therefore mainly on the categorization of welding processes. However, due to this strict and specific focus, the ontology is not generic enough to provide a common basis for describing information and findings about mechanical joints. In addition, some ontology projects aim at providing a knowledge representation, which integrates design and manufacturing knowledge. Thereby, feature-based approaches on the basis of CAD technologies dominate the field. Kim et al. [2] proposed an approach for ontology-based assembly design and information sharing. The focus of their work is on describing assembly design and thus the engineering, spatial, assembly and joining relations of products. Additionally, the scope is mainly on the definition of features and therefore provides an analysis of terms and representations within the domain of assembly design. However, it remains unclear whether their work contribute to other domain ontologies with similar scope, such as design ontology (DO) [3]. Li et al. [4] introduce an ontology-based approach for manufacturability verification and knowledge reuse. The introduced framework is based on the core product model [5] as well as on the standard ISO 1303 AP224 and aims at a rule-based manufacturing verification using Semantic Web Rule Language (SWRL). Thereby, a design ontology describing geometrical features are mapped to manufacturing processes defined in a manufacturing ontology. However, the ontology is mainly based on standard CAD features (e.g. holes, cylinders) and their corresponding manufacturing process (e.g. drilling, milling, welding), but lacks a deeper characterization of the concept of mechanical joining. In summary, previous works showed already high potentials for the application of ontologies in mechanical domains. Hence, within this contribution a specific and novel ontology (in the following referred as JOINology) is presented as a first step to overcome the lack of a formal representation for reusing and sharing of knowledge in the domain of mechanical joining. Thereby, the focus is on facts regarding the mechanical joint as central object of interest and on aligning information to this object concerning joining possibility, suitability and safety using clinching and pin joining as an example.

\section{Research questions}

Given the lack of knowledge databases in the field of mechanical joining, this contribution aims to introduce a method for the provision of cross-domain knowledge using ontologies in order to answer three research questions (RQ). Following on the detailed description of the methodical background involving a brief overview of ontologies and mechanical joining processes, the subsequent section answers the question, whether a suited informal domain theory exists to structure knowledge of mechanical joining (RQ1). This includes the identification of relevant terms and definitions in the field of mechanical joining from norms and standard references. Then, the investigation of initial scenarios concerning the sharing, reuse and searching of knowledge in mechanical joining defines the scope and purpose of the developed ontology. Therefore, the formulation of so-called competency questions (CQs), which are used as a kind of requirements specification within ontology engineering, enable the testing of the system. By comparing the underlying domain theory and the identified scenarios for sharing and reuse of knowledge, the following section uses clinching as an example to test how comprehensive and applicable the generated knowledge representation already is (RQ2). Based on these results, the subsequent chapter evaluates whether the setup of an ontology is feasible and which further developments are necessary to provide and support the efficient sharing and reuse of cross-domain knowledge in the field of mechanical joining (RQ3).

\section{Methods}

The following sections provide a detailed overview of the applied methods for the setup of an ontology and the definition of formal queries based on pre-defined competency questions. Furthermore, the cold-forming processes clinching and pin joining are explained in more detail as representatives for the field of mechanical joining processes.

\subsection{Ontologies and knowledge reuse}

According to Gruber [6] an ontology is an "explicit specification of a conceptualization". In other words, an ontology can be used to formally describe and structure the knowledge of a domain in a human- and machine-readable way. In the 
context of knowledge sharing and reuse an ontology provides schema-level knowledge that can be used to structure and query a knowledge base (KB). Since humans usually communicate in natural language and thus within scientific domains, norms or standard references mainly offer taxonomies or glossaries to define terms of interest and enable a common understanding between all participants. This can be seen as a semiformal conceptualization, since a humanunderstanding is possible. However machine-processing of knowledge is difficult within a natural language representation. Therefore, modeling knowledge for the purpose of reuse and sharing in a formal ontology-language, such as OWL [7], enables an explicit specification of the already loosely existing conceptualization in combination with a machine-processability. In domains like bioinformatics, ontologies are widely used for knowledge structuring, data integration and decision support systems [8]. A well-known ontology is the gene ontology (GO) [9], which provides broadly accepted vocabulary for annotating gene product data from different databases and experiments. Moreover, exploiting ontologies for accessing and reusing knowledge has already been pursued in other engineering domains, such as tribology. For this purpose, the authors in [10] introduced the tribAIn ontology as a schema for structuring and reusing knowledge from tribological experiments. Within the domain of material science, the European Materials Modelling Ontology (EMMO) [11] aims to provide a representational ontology for materials modelling and characterization. Ontologies take a central role in the context of the Semantic Web, since they are suited for data integration and sharing not only on human-level or human-machinecommunication, but also in machine-machine-interaction. A common application is semantic annotation of unstructured documents [12]. The semantic enrichment of unstructured content enables the application of semantic search, which means searching beyond keywords and thus improving the retrieval of queries. Since formal ontology languages are usually based on description logics [13], a distinction between terminological and assertional knowledge within an ontology-based KB (see Fig. 1) exists. The ontology provides terminological, and thus generic knowledge, which structures the underlying assertional object knowledge. For this purpose, ontologies are formulated in triple notation, meaning they can be visualized as a directed graph with objects linked by named relations.

\subsection{Mechanical joining processes}

For the subsequent introduction of the generated knowledge representation the mechanical joining processes pin-joining and clinching are used as an example. For this reason, both procedures are described in more detail in the following sections.

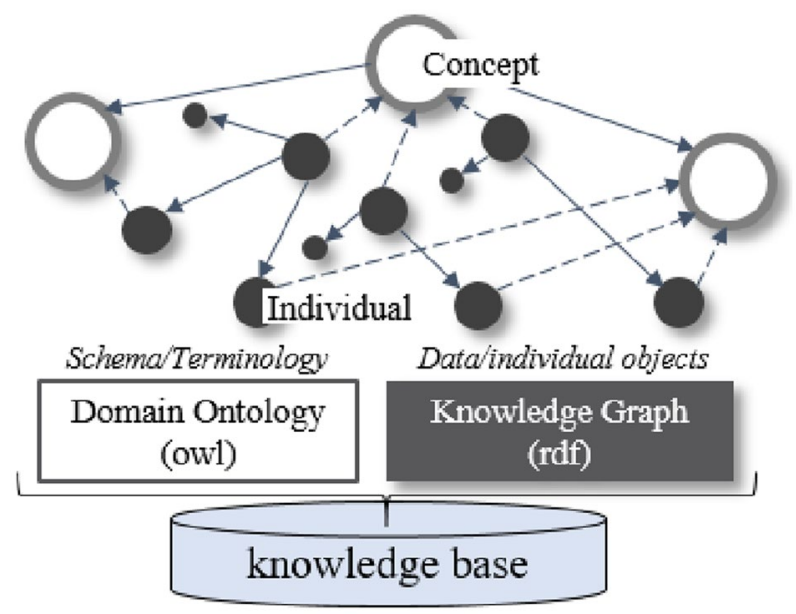

Fig. 1 Schematic architecture of a semantic knowledge base

\subsubsection{Clinching}

Conventional clinching (classified as "joining by forming" (DIN 8593-5)) is a mechanical joining technique, which is becoming increasingly important in complex multi-material lightweight designs, such as in car body parts, and is thus a highly relevant cold-forming joining process. In clinching, tools are used to join two or more overlapping sheets, tubes or profiled parts by cold forming without auxiliary joining parts [14].

The joining process can be divided into four different stages (Fig. 2). Three different characteristic tools are required for the clinching process-a punch, a die and a blank holder. In the first stage, a blank holder fixes the sheets or parts to be joined by applying a force in the joining direction. In the second stage, the punch begins a continuous working stroke to press the punch layer sheet into the die sided sheet by cold forming. This results in combined penetration of the joining partners. The subsequent compression leads to extrusion, resulting in an inseparable joint. The connection is form-fit, force-fit and in individual cases, there is also a material-fit, between components made of different, even higher-strength materials [15]. In the third phase the maximum punch stroke is reached. The clinch connection is finally released in the fourth phase by the return stroke of the punch as well as the hold-down device, resulting in elastic spring back. In this process, the joinability of material-geometry combinations is limited by the formability of the materials due to the high plastic strains that occur during the process [16]. The joining zone can be described by several parameters (see Fig. 3). Beside the thicknesses of the punch layer sheet $t_{1}$ and the die layer sheet $t_{2}$ the inner diameter $d_{i}$ and the outer diameter $d_{0}$ are determined by the joining setup. In addition, the button height $h$ is often specified. These parameters are primarily influenced by the 
Fig. 2 Tool design and sequence for clinching with closed die [14]

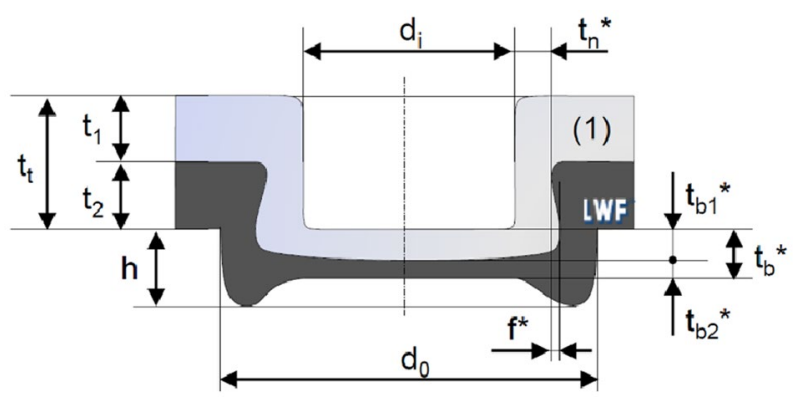

Fig. 3 Geometric parameters of a clinch joint $[15,16]$

tool and sheet metal properties. For a more detailed investigation of the joining process, the geometry of the resulting clinch point is taken into account. Therefore, micrographs are prepared and analysed. Three characteristic geometric parameters (bottom thickness $t_{b} *$, interlock $f^{*}$ and neck thickness $t_{n}{ }^{*}$ ) are considered relevant to quality aspects of the joint [16].

The interlock $f^{*}$ and the neck thickness $t_{n}{ }^{*}$ have a direct influence on the failure modes of the clinch joint and thus on the load-bearing capacity of the joint [17]. Both correlate with the bottom thickness $t_{b} *$, which can be utilized as a non-destructive evaluable, quality determining parameter if the tool and process parameters are known. The bottom thickness lies in a range of approx. 20-50\% of the total work piece thickness $t_{t}$. The blank holder force and the surface condition of the parts to be joined additionally influence the formation of the joints. In addition to the investigation of the clinch point forming during the joining process, destructive analyses and force-displacement values are used. In strength testing, a distinction is usually made between quasi-static and dynamic testing. The max. shear and head tensile as well as peel tensile forces are usually determined for both types of stress [16].

\subsubsection{Pin joining}

In the scope of this paper, pin-joining is used as an example for the field of mechanical joining since the technique is a comparatively new joining method with only less reliable design principles and is therefore representative for the importance of knowledge reuse and sharing. In general, the pin structure is characterized as a small rod protruding from the surface of one joining partner, which is embedded in the second joining partner. Thereby, the pin usually has a diameter and height of more than $1 \mathrm{~mm}$ showing an aspect ratio of height to diameter typically larger than one. Mostly, pins have a cylindrical or rectangular cross section [18] depending on the manufacturing process or desired mechanical properties. Pin structures for joining dissimilar materials are produced by various manufacturing processes, which can be primarily categorized as molding [19], additive [20] and formative [21]. Pin joints mostly are known for metalmetal joints [22] as well as metal-composite (thermoset [23] and thermoplastic [21] matrix systems) joints. Thereby, two main process routes are established:

- The pin intersection is directly pressed into the second joining part. For this purpose, the thermoplastic matrix often has to be heated in advance [24, 25].

- Or a two stage process is applied, where the pin is pushed through a pre-manufactured hole and is afterwards caulked to create an undercutting form-fit [20,21].

In comparison to other mechanical joining processes, pin joining avoids the destruction of load bearing fibers and thus leads to potentially increased joint strengths. Additionally, similar to clinching, no weight is added through auxiliary elements, which offers high potentials for the application of the procedure in lightweight designs. Furthermore, for direct pin pressing and partly pin caulking, the elimination of the need for precisely manufactured bolt holes leads to cost-efficient and fast manufacturing processes. Moreover, through the adaption of the pin geometry it is possible to react to changes in the base materials or requirements on the joint leading to a versatile joining technology. As an example, Fig. 4 illustrates an overview of the individual pin joining processes. 
Direct pin pressing

Pin caulking

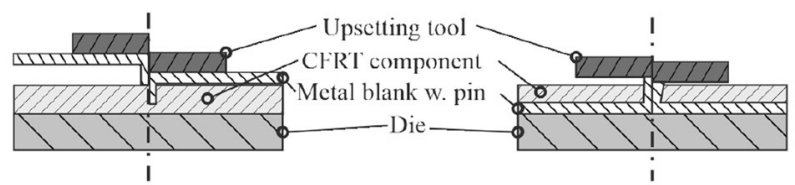

Fig. 4 Illustration of both pin joining processes

\section{Development of an ontology within the domain of mechanical joining}

\subsection{Purpose and scope}

A first step in ontology engineering is the determination of initial scenarios (cf. [26]) and competency questions, which represents the areas to be covered by the knowledge base. Since the field of mechanical joining involves several domains, the development of an ontology considers three main areas of production (joining possibility), material (joining suitability) and design (joining safety). Then, the already mentioned formulation of competency questions enables the informal specification of requirements for the ontology and the following testing and evaluation of the system coverage. The subsequent overview determines the three use-case areas defined by superordinate CQs given by domain experts. Those question are formulated due to issues of interest within the domain of mechanical joining and thus determine the three main areas process, component and application of the JOINology:

- Joining possibility The creation of multi-material connections requires not only the selection of suitable joining techniques but also the determination of an efficient assembly and joining order in combination with the adherence to design principles. This involves for example the consideration of minimal possible joint distances or the accessibility of the joint. Example CQs Is it possible to join the materials using the chosen tool set? What is the influence of changing joint distances on the resulting feasibility of the entire connection within a joining process chain?

- Joining suitability Beside the definition of the joining possibility, the generated knowledge base has to be able to provide reliable responses on queries about which design and material investigations are already implemented in the ontology. Example CQs Which material and sheet combination can be joined under a certain blank thickness ratio? What are suitable material properties (e. g. ductility or tensile strength) to achieve a certain material flow?
- Joining safety To avoid the failure of the joint connection the reliable design and construction of joints is one of the most crucial steps. This includes for example the investigation of impact values on the joint strength caused by material and process variations. Example CQs How do pre-processes such as a deep drawing change the material behavior? Which types of load can be applied for a certain material and sheet thickness combination?

The introduced competency questions represent examples for the overall scope and purpose of the generated ontology.

\subsection{Domain theory and concepts within the domain of mechanical joining}

Even if a formal specification of the domain of mechanical joining is not available, different norms (e.g. [27]) and standard references (e.g. [28]), provide some kind of domain theory including taxonomies and properties of joining processes. On this basis a formal specification of the domain is established following a middle-out approach [26] meaning the definition of the most important concepts of the domain before generalizing and specifying them. In the following, we will use the CamelCase notation for concepts and visualize excerpts of the ontology as a directed graph. From an ontological perspective the object of interest is a specific mechanical joint, which is linked to information from designing, simulation, testing, production and application. Figure 5 shows an excerpt of the JOINology with focus on a clinched joint and the concepts referring to the main areas component, application and process. Following the taxonomy provided in [28] the clinched joint can be described as a sheet metal joint which consists of the joining of sheet metals without additional joining element. The taxonomic relationship is modeled as an is-a relation between the concepts ClinchedJoint, SheetMetalJoint and MechanicalJoint. The following axiom (notations base on the Description Logic) further specifies the clinched joint referring to the classification by usage of an additional joining element:

\section{SheetMetalJoint $\sqcap \neg \exists$ hasJointPart.JoiningElement $\sqcap \exists$... ...hasJointPart.SheetMetal $\sqsubseteq$ ClinchedJoint}

Since the process configuration of manufacturing a joint can vary, the concept Clinching is assigned to provide further information of the procedure and tools used within the joining process. As an example, according to [27, 28], the setup of the procedure depends on whether the clinching involves a cut section or not (see Fig. 5). Therefore, the JOINology enables a generic description of tools (here: clinching with or without cutting section is assigned to the tool concepts Die, BlankHolder and Stamp). Further information about how individual tools can be described in more detail by concrete 


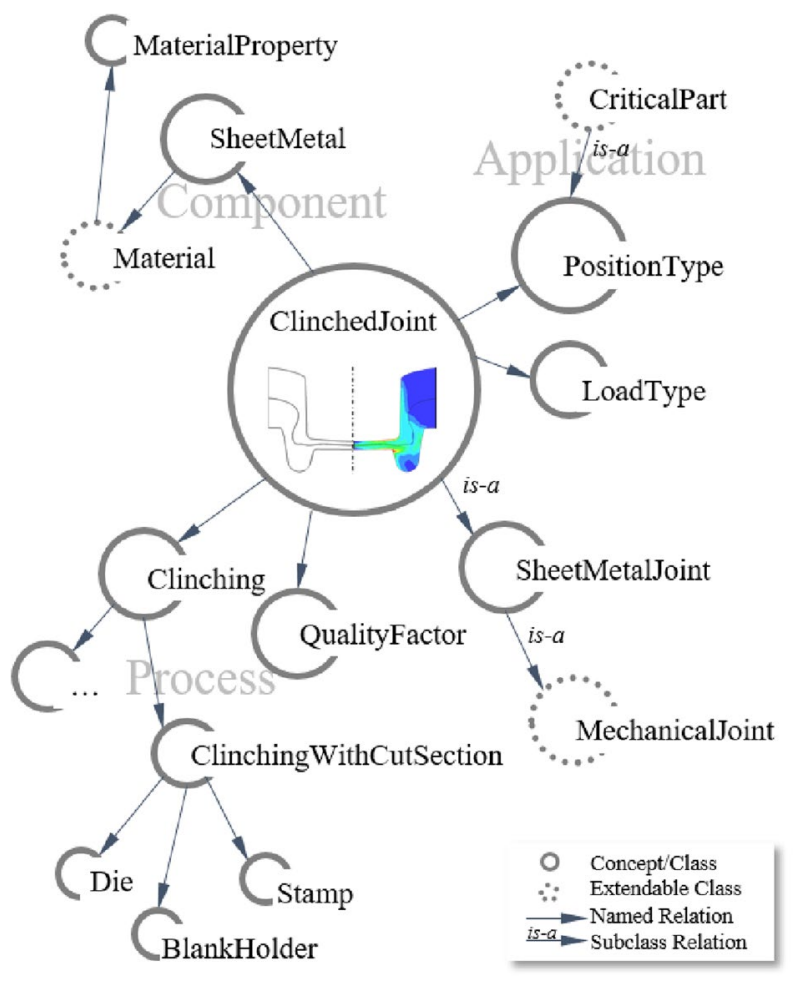

Fig. 5 Core concepts and relations within the JOINology focused on clinched joints

parameters, such as defining the diameter of die or punch used for manufacturing a specific clinched joint, will be demonstrated in Fig. 7. Besides the formerly mentioned is$a$ relation, which is always used for building hierarchical and thus inheriting linkages between objects, named relations are used to describe directed relationships. For instance, the following notation shows that a clinched joint has some quality factors, like the interlock and the neck thickness:

\section{ClinchedJoint $\sqsubseteq \exists$ hasQualityFactor.InterlockThickness $\sqsubseteq \exists$ hasQualityFactor.NeckThickness $\cdots$}

Since some geometrical characteristics of the connection have a quality-relevant impact on specific joint properties, such as on the mechanical strength, the relation called Regulates offers the opportunity to sign whether an increase of the concerned parameter has a positive (positivelyRegulates) or a negative (negativelyRegulates) influence on the resulting joints' strength. As an example, the following two axioms state that the neck thickness has a positive influence on the shear strength while the thickness of the interlock positively influences the tensile strength:

NeckThickness $\sqsubseteq$ ヨositivelyRegulates.ShearStrength InterlockThickness $\sqsubseteq \exists$ positivelyRegulates.TensileStrength

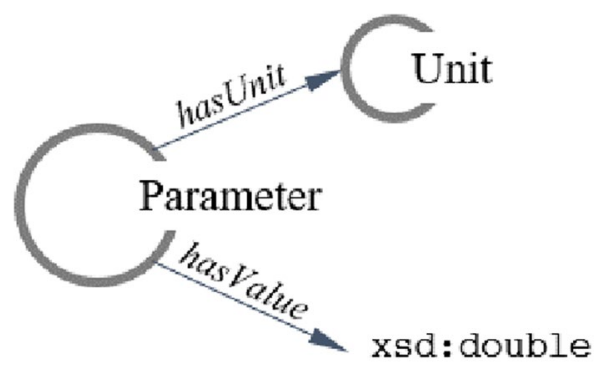

Fig. 6 Parameter description by the triple parameter hasUnit unit and parameter hasValue xsd:double

Another important pattern used in the JOINology is the description of parameters in combination with a value and unit. A value must be of datatype xsd:double and the unit is assigned by the relation hasUnit to a parameter (see Fig. 6).

Since this contribution discussed the interdisciplinary character of mechanical joining already, some of the concepts within the core ontology are explicitly intended for extention. Those are signed within Fig. 5 with the dotted circles. For instance, the concept of Material relies on an own domain theory. Thereby, material can be seen as a product of chemical synthesis or simply as a substrate, depending on the application and knowledge domain. The concept of a critical part is currently dealt as a single entity. This concept can be further extended by partly adopting for instance concepts and relationships from the DO [3], depending on the need for a detailed description of geometry or system structures. However, those concepts are not in the focus of the JOINology, which is intended to structure information related to a mechanical joint and, especially within this contribution, to a clinched and pin joint.

\section{Results}

The subsequent chapters introduce the setup of the knowledge representation in more detail using pin-joining and clinching as an example. For this purpose, the excerpt of the knowledge base is demonstrated on the latter procedure while the testing of the ontology considering example queries is demonstrated for both methods.

\subsection{Use-case: instantiation of pin and clinched joints}

To ensure a high reliability of the connection not only the identification of a fitting mechanical joining technique but also the selection of suitable process parameters are crucial. This is often based on the try-and-error iterations of experimental studies and the involvement of expert knowledge 
Fig. 7 Overview of the clinching and pin joining process in combination with resulting quality-relevant joint properties

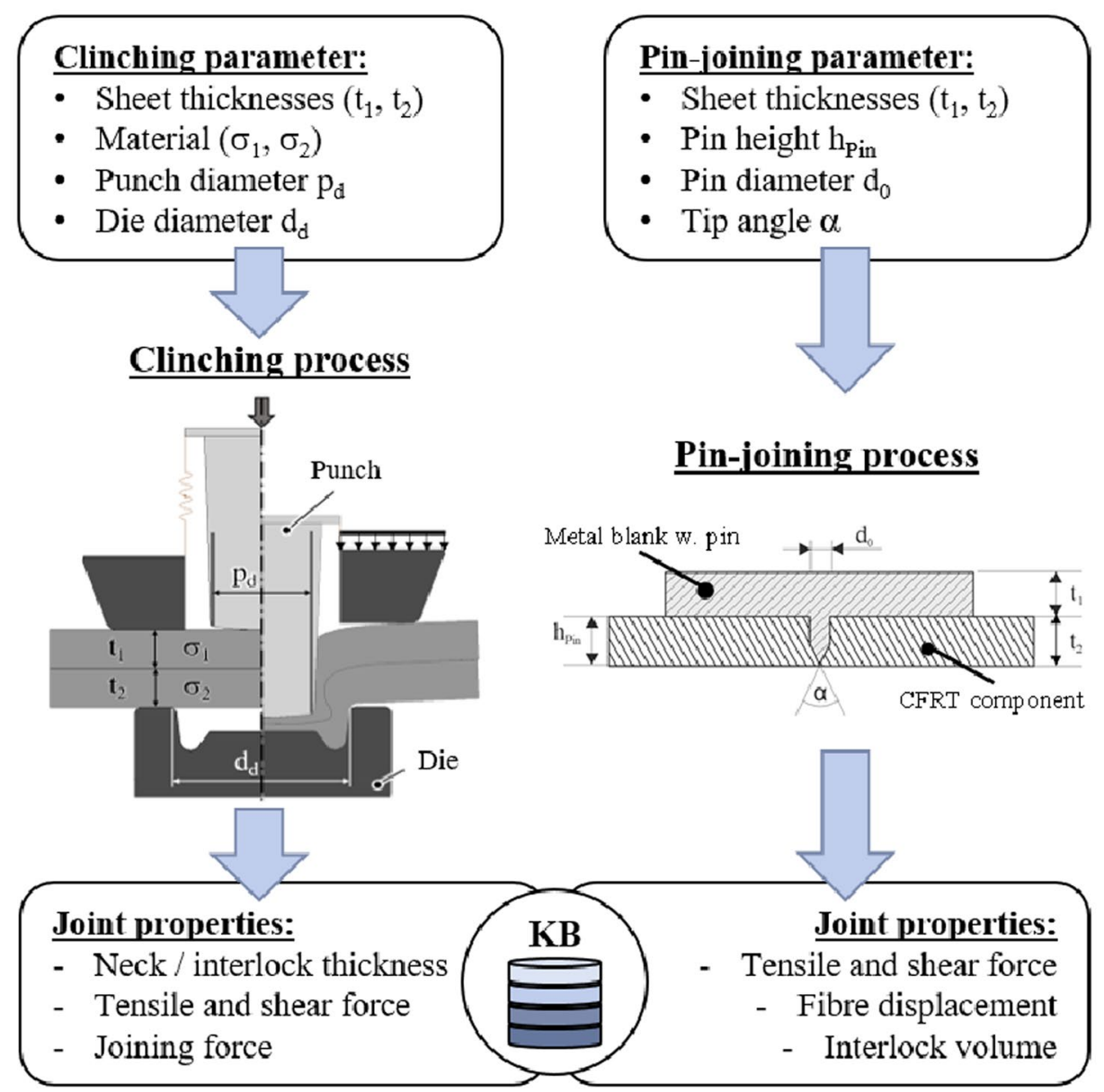

until a certain level of joint properties is reached. As this mainly implies the time- and cost-intensive inclusion of resources the availability and reuse of already existing knowledge is of high importance. Regarding the clinching of sheets (see Fig. 7), the achievement of a sufficient joint strength against tensile and shear loading is dependent on the selection of several blank and process parameters, such as the sheet thicknesses or the diameters of punch and die. Therefore, the measurement of quality relevant geometrical joint characteristics, such as the neck and interlock thickness, enable to evaluate whether the connection is suitable for a specific type of load or not. In comparison, the pin joints' resistance against shear loading is mainly dependent on the initial selection of geometrical characteristics, such as the pin diameter or tip angle. However, if a new scenario (e. g. changing materials) has to be taken into consideration, the resulting joints of both methods do not necessarily show equal characteristics and properties using previously defined joining settings. Thus, a sufficient reliability on the connection can no longer be guaranteed and the design and dimensioning process starts again.

This use-case was chosen as the investigation of clinch and pin joints involves the appropriate coordination between the core concepts Process, Component and Application and is thus excellently suited to test and evaluate the applicability of JOINology.

\subsection{Querying JOINology}

Based on the use-case data, instances are created using the ontological schema to structure and semantically describe the single data objects. The following excerpt of the KB shows triples describing facts about a clinched joint named CJ_001. The excerpt is written in Turtle-Syntax [29], which is a common serialization format for graph databases and thus also used for ontology-based KBs. The prefix jo is used here for the IRI (Internationalized Resource Identifier) of the JOINology, while the prefix $d$ signs the IRI of the data objects from the use-case (see the header within the Turtle representation). 
PREFIX rdf: 〈http://www.w3.org/...-rdf-syntax-ns\#> PREFIX xsd: 〈http://wwW.w3.org/2001/XMLSchema\#> PREFIX jo: 〈http://ktmfk.de/ontology/JOINology\#> PREFIX d: 〈http://ktmfk.de/ontology/JOINology/data\#〉

\begin{tabular}{|c|c|c|}
\hline d:CJ_ø01 & $\begin{array}{l}\text { rdf: type } \\
\text { jo:hasJointPart } \\
\text { jo: hasJointPart } \\
\text { jo: hasParameter } \\
\text { jo: hasParameter }\end{array}$ & $\begin{array}{l}\text { jo:ClinchedJoint; } \\
\text { d:SH_ST001_1; } \\
\text { d:SH_ST001_2; } \\
\text { d:HTS_001; } \\
\text { d:UC_001. }\end{array}$ \\
\hline d:SH_ST001_1 & $\begin{array}{l}\text { rdf:type } \\
\text { jo:hasMaterial } \\
\text { jo:hasParameter }\end{array}$ & $\begin{array}{l}\text { jo:SheetMetal; } \\
\text { d:EN_AW-6014; } \\
\text { d:Thickness_ST001. }\end{array}$ \\
\hline \multicolumn{3}{|l|}{$\ldots$} \\
\hline d:HTS_001 & $\begin{array}{l}\text { rdf:type } \\
\text { jo:hasValue }\end{array}$ & $\begin{array}{l}\text { jo:HeadTensileStrength; } \\
\text { "1700.0"^^xsd:double. }\end{array}$ \\
\hline d:UC_001 & $\begin{array}{l}\text { rdf:type } \\
\text { jo: hasValue } \\
\text { jo: hasUnit }\end{array}$ & $\begin{array}{l}\text { jo:Interlock; } \\
\text { " } 0.3 \text { J"^sd:double; } \\
\text { jo:Millimeter. }\end{array}$ \\
\hline
\end{tabular}

The instance CJ_OO1 is described as of the type ClinchedJoint (CJ_001 rdf:type ClinchedJoint). In addition, some more facts about the joint are known from the use-case data, thus this instance is linked to other objects within the KB, e.g. CJ_O01 hasJointPart SH_ST001_1. This relates the clinched joint $C J \_001$ to one of the two joint components, such as the sheet metal SH_STO01_1. Moreover, this instance is linked to further objects describing its material or the geometric parameters of the sheet metal. Some of the objects CJ_O01 have a discrete value (e.g. HTS_001 hasValue "1700.0"). In this way, every triple provides more information about an object within the KB and through interlinking the objects a knowledge graph is generated using the ontology as semantic schema.

For querying the data, the SPARQL 1.1 query language [29] is used. The generated query (see Fig. 8) retrieves all clinched joints within the knowledge base, which can resist a certain tensile strength (FILTER (?TensileStrength>1000)). Since the adequate formation of quality-relevant geometrical joint characteristics, such as the neck and interlock thickness (?THKNeck, ?THKInterlock), and thus a sufficiently high tensile strength depend on the selection of suitable process and material parameters [17], the query also retrieves those values from the knowledge base (?MATUpperSheet, ?MATLowerSheet, ?THKUpperSheet, ?THKLowerSheet). The resulting output of the query is shown in Fig. 9.

One can see, that only two clinched joints were filtered from the data through the example query. For instance, CJ_001 provides a tensile strength of $1700 \mathrm{~N}$ using a similar material and sheet thickness combination (EN AW-6014 $\mathrm{T} 4,2.0 \mathrm{~mm}$ ). Thus, the product designer can choose between
PREFIX owl: <http://wwlw3.org/2062/07/owl\#> PREFIX rdf: 〈http://www.w3.org/1999/02/22-rdf-syntax-ns\#> PREFIX rdfs: 〈http://www.w3.org/2000/01/rdf-schema\#> PREFIX jo: 〈http://ktmfk.de/ontology/2021/8/JoINology\#〉

SELECT ?ClinchedJoint ?THKUppersheet ?THKLowersheet ?MATUpperSheet ?MATLowerSheet ?THKNeck ?THKInterlock ?Tensilestrength WHERE \{

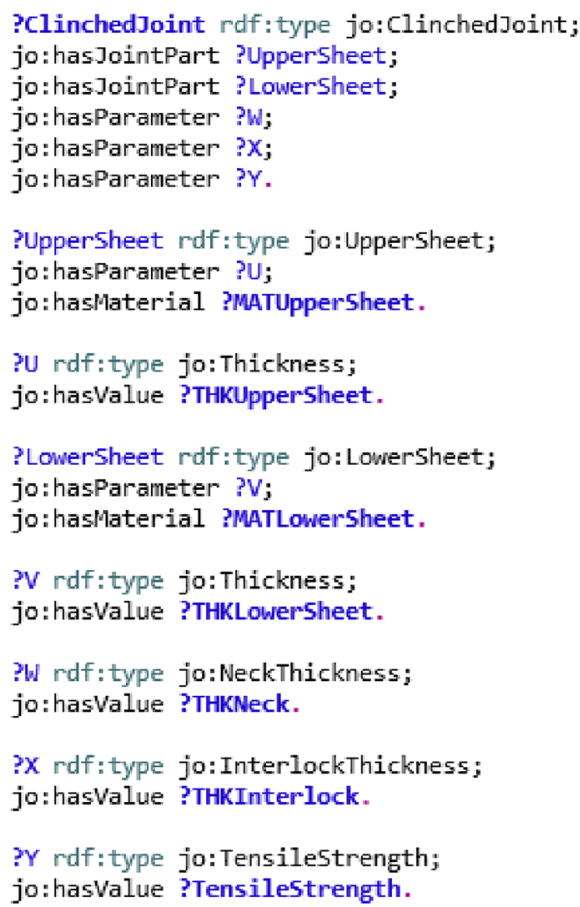

Fig. 8 Example query for filtering a specific tensile strength

these joint configurations to identify the best-fitting solution for an individual use-case scenario.

As a further example, the concept of QualityFactors can also be exploited to retrieve only the important factors for a respective mechanical joint. The query in Fig. 10 selects the quality factors of the mechanical joints within the data as well as the particular strength for the individual joint.

The influence of quality factors can be seen for instance by comparing the two pin joints (see Fig. 11) PJ_OO2 and PJ_003, which have the same tip angle, but different pin diameters.

Therefore, according to [30], an increased pin diameter leads to improved maximum shear forces. This can be explained with the failure mechanism: the pin first bends in the direction of the shear force and is consequently, due to the altered flux of force, pulled out of the CFRT component. An increased diameter leads to a higher resistance against bending and consequently to higher maximum forces.

In comparison, a steeper tip angle (see PJ_003 and PJ_004) leads to decreased reaction forces as a smaller 
Fig. 9 Output of first example query

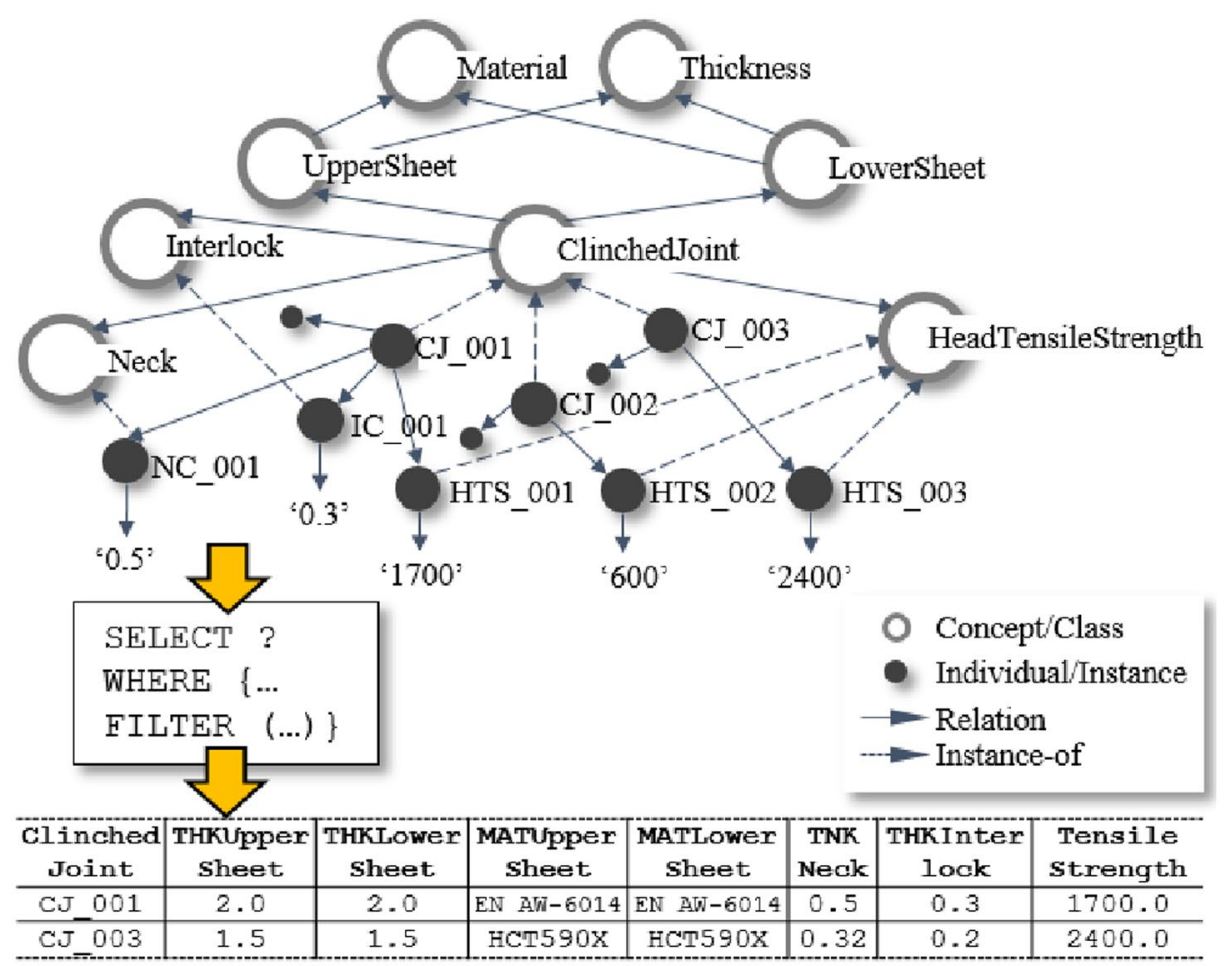

PREFIX owl: 〈http://www.w3.org/2002/07/owl\#>

PREFIX rdf: <http://www.w3.org/1999/02/22-rdf-syntax-ns\#> PREFIX rdfs: לhttp://wusw.w3.org/2000/01/rdf-schema\#s

PREFIX jo: 〈http://ktmfk.de/ontology/2021/8/JOINology\#>

SELECT ?Joint ?Strength ?SValue ?QFactor ?QValue WHERE \{

?QFactor rdf:type jo:QualityFactor;

jo:hasvalue ?QValue.

?Joint ndf:type jo:MechanicalJoint;

jo:hasParameter ?QFactor;

jo:hasParameter ?Strength.

?Strength rdf: type jo:Mechanicalstrength;

\} jo:hasValue ?SValue.

Fig. 10 Example query for filtering quality factors

cylindrical section results in a reduced transmission of the shear forces. Consequently, the tip angle can be assumed as a draft angle, which leads to a earlier pin extraction and decreased maximum shear forces.

In summary, the presented queries showed examples for the feasibility of the generated ontology in the field of mechanical joining. However, these illustrations introduces only a strongly simplified example of a possible knowledge graph representation. To ensure a high applicability of the ontology it is mainly necessary to integrate a sufficient amount of data into the knowledge database. As shown, the data can be filtered and combined by desired conditions and retrieved in a concise way. Since the ontological schema offers explicit semantics, data interoperability can

\begin{tabular}{|c|c|c|c|c|}
\hline Joint & strength & svalue & QFactor & Qvalue \\
\hline \multirow[t]{2}{*}{ CJ_001 } & \multirow{2}{*}{$\begin{array}{c}\text { HeadTensilestr } \\
\text { ength_001 }\end{array}$} & \multirow[t]{2}{*}{1700} & Interlock_001 & 0.30 \\
\hline & & & NeckThickness 001 & 0.50 \\
\hline \multirow[t]{2}{*}{ CJ_002 } & \multirow{2}{*}{$\begin{array}{c}\text { HeadTensilestr } \\
\text { ength_002 }\end{array} \mid$} & \multirow[t]{2}{*}{600} & Interlock_002 & 0.15 \\
\hline & & & NeckThickness 002 & 0.25 \\
\hline \multirow[t]{2}{*}{ CJ_003 } & \multirow{2}{*}{$\begin{array}{c}\text { HeadTensilestr } \\
\text { ength_003 }\end{array}$} & \multirow[t]{2}{*}{2400} & Interlock_003 & 0.20 \\
\hline & & & NeckThickness 003 & 0.32 \\
\hline \multirow[t]{2}{*}{ PJ_001 } & \multirow{2}{*}{$\begin{array}{c}\text { ShearTensilest } \\
\text { rength_001 }\end{array}$} & \multirow[t]{2}{*}{473} & PinDiameter_001 & 1.50 \\
\hline & & & Tipangle 001 & 90.0 \\
\hline \multirow[t]{2}{*}{ PJ_002 } & \multirow{2}{*}{$\begin{array}{c}\text { ShearTensilest } \\
\text { rength_002 }\end{array}$} & \multirow[t]{2}{*}{313} & Pindiameter_002 & 1.5 \\
\hline & & & TipAngle_002 & 45.0 \\
\hline \multirow[t]{2}{*}{ PJ_003 } & \multirow[t]{2}{*}{$\begin{array}{c}\text { ShearTensilest } \\
\text { rength_003 }\end{array}$} & \multirow[t]{2}{*}{226} & PinDiameter_003 & 1.0 \\
\hline & & & TipAngle_003 & 45.0 \\
\hline \multirow[t]{2}{*}{ PJ_004 } & \multirow{2}{*}{$\begin{array}{c}\text { ShearTensilest } \\
\text { rength_004 }\end{array}$} & \multirow[t]{2}{*}{372} & PinDiameter_004 & 1.00 \\
\hline & & & TipAngle_004 & 90.00 \\
\hline
\end{tabular}

Fig. 11 Output of second example query

be accomplished and thus data from different sources can be queried and retrieved over the same ontological schema. 


\section{Discussion}

Within this contribution an ontology was introduced for the purpose of reuse and sharing mechanical joining knowledge. Therefore, in Sect. 5.2 the main concepts were presented and visualized as a directed graph (see Fig. 5). The ontology was built based on the already available domain theory from norms and other standard literature. The structure of the ontology was highly influenced by the use-cases described within Sect. 5.1, thus the concepts and relations were modeled intended for comply the competency questions for joining possibility, suitability and safety. Therefore, the first question (RQ1) introduced in Sect. 3 can be confirmed, since a semiformal conceptualization from the domain of mechanical joining is already available and was used to formally specify the main concepts. Especially taxonomies and the definition of tools for different joining procedures are a basis to model a representation suited to answer the CQs from Sect. 5.1. This procedure of comparing the already existing conceptualization in standard literature with the defined use-cases refers to RQ2. The JOINology is currently built in a middle-out fashion, since there exists no formal and common knowledge model to serve the information needs of joining possibility, suitability and safety. Beginning with the most central objects of the domain, the mechanical joint itself, existing informal taxonomies were related in a topdown manner. Nevertheless, further investigation is needed in the bottom-up direction, which means analyzing the need for alignment and the role of the ontology to middle-level or upper-level ontologies. Since these ontologies deal with generic ontological concepts independent of a domain, they serve as a schema for providing interoperability of different domain ontologies. As already mentioned, mechanical joining is a highly interdisciplinary domain and thus theories from other domains are needed as well. As signed in Fig. 5 with the dotted circles some concepts need further extension. For instance, material models (cf. the EMMO project mentioned in Sect. 4.1) from the domain of material science can be linked to the JOINology to get more information. Moreover, the inclusion of additional mechanical joining processes provides a comprehensive knowledge base for the comparison and selection of different procedures based on their joining properties and application possibilities. Especially, the implementation of the presented pin joining process offers a further opportunity to join dissimilar materials without auxiliary joining parts. However, since product designers can only rely on less design principles, the sharing and reuse of already existing knowledge can lead to a faster and cost-reducing dimensioning of pin structures. This becomes particularly important when the main process route differs between the joining of a metal-metal or a metal-composite connection. The major difference between both material combinations from a process perspective is that metal-metal joining is typically conducted at room temperature which leads to a cold forming of both joining partners resulting in the formation of undercuts. In contrast, for the joining of metal-composites, the matrix is either uncured (thermoset based) or in a molten state (thermoplast based) which leads to no cold forming of the pin. Consequently, no undercuts are formed during the joining process and, if necessary for the joint, need to be considered in the pin manufacturing process.

In summary, the implementation of additional mechanical joining processes enable the product designer to choose between several methods and thus to identify the best-fitting solution (e.g. tool and process configuration) for a certain use-case by querying the ontology. Therefore, RQ3 can only partly be answered, since not all needed extensions could be identified within the scope of this contribution. However, the ability to expand is an important opportunity and coincidentally a major weakness of ontological models. An ontology can relatively easily be extended, and even other existing ontologies can be mapped, thus it provides a flexible representation. On the other hand, Hoekstra [31] argues this can lead to a violation of the ontological commitment. As described in Sect. 4.1 an ontology is built as an explicit agreement about a conceptualization within a domain, thus models from other domains can provide a different view and understanding of objects within an ontology. Therefore, extensions must be made carefully, but are otherwise a great opportunity to link and retrieve cross-domain knowledge needed for mechanical joining. The use-case shows that the ontological schema is able to capture knowledge for the clinched and pin joints introduced within Sect. 6.1. The instantiation here is made manual for the purpose of demonstration. Otherwise, maintaining a KB manually is time-consuming as well as error-prone. Resulting, procedures for manual and automatic contribution to a semantic $\mathrm{KB}$ within the domain of mechanical joining are vital for long-term use and reliability. As an example, the authors in [32] already argued for the domain of tribology, that different data sources in structured and unstructured formats need several alignment strategies to be integrated in a semantic KB. For this purpose, successfully applied implementations from the domain of biomedicine and bioinformatics can function as role models.

\section{Conclusion and outlook}

Summary This contribution presents an ontology for the provision of cross-domain knowledge about mechanical joining processes. Therefore, the setup of the knowledge representation bases on the formalization and standardization of already available domain theory from norms and 
other standard literature. Additionally, the structure of the ontology and the phrase of competency questions rely on concepts and relations concerning to the main areas of production (joining possibility), material (joining suitability) and design (joining safety). Subsequently, the formulation of formal queries (SPARQL1.1 query language) enable to test and evaluate the applicability of JOINology using the clinching and pin joining of sheets as an example. In summary the setup of an ontology in the field of mechanical joining processes shows the following results:

- It is possible to structure knowledge of mechanical joining within an ontology since a semiformal conceptualization from the particular domains is already available (norms and standard literature) and thus this enables the formally specification of main concepts and relations.

- However, due to a high multidisciplinarity within the domain of mechanical joining the embedding of further and already existing ontologies can extend the applicability of JOINology.

Outlook As already mentioned, mechanical joining is a highly interdisciplinary domain involving a great amount of data and information, which have to be efficiently linked in the knowledge representation. The more input is integrated into the ontology, the more powerful it becomes. Therefore, the careful implementation of already existing models from other domains, such as material models, and the inclusion of further mechanical joining processes, such as riveting, can provide a more comprehensive view and understanding of objects within the ontology in the future.

Acknowledgements This work was Funded by the Deutsche Forschungsgemeinschaft (DFG, German Research 635 Foundation)-TRR 285-Project-ID 418701707.

Author contributions CZ: conceptualization, methodology, formal analysis, investigation, writing-original draft, visualization, PK: conceptualization, methodology, software, formal analysis, investigation, writing-original draft, visualization, JP: methodology, writing-original draft, CRB: methodology, writing-original draft, visualization, MB: formal analysis, writing-review and editing, DD: formal analysis, writing-review and editing, GM: formal analysis, writing-review and editing, SW: formal analysis, writing-review and editing, BS: conceptualization, methodology, formal analysis, writing-review and editing, supervision

Funding Open Access funding enabled and organized by Projekt DEAL.

Data availability statement Not applicable.

Code availability Not applicable.

\section{Declarations}

Conflict of interest The authors have no conflicts of interest to declare that are relevant to the content of this article.

Open Access This article is licensed under a Creative Commons Attribution 4.0 International License, which permits use, sharing, adaptation, distribution and reproduction in any medium or format, as long as you give appropriate credit to the original author(s) and the source, provide a link to the Creative Commons licence, and indicate if changes were made. The images or other third party material in this article are included in the article's Creative Commons licence, unless indicated otherwise in a credit line to the material. If material is not included in the article's Creative Commons licence and your intended use is not permitted by statutory regulation or exceeds the permitted use, you will need to obtain permission directly from the copyright holder. To view a copy of this licence, visit http://creativecommons.org/licenses/by/4.0/.

\section{References}

1. Saha S, Usman Z, Li WD, Jones S, Shah N (2019) Core domain ontology for joining processes to consolidate welding standards. Robot Comput Integr Manuf 59:417-430. https://doi.org/10. 1016/j.rcim.2019.05.010

2. Kim KY, Manley D, Yang H (2006) Ontology-based assembly design and information sharing for collaborative product development. Comput Aided Des 38(12):1233-1250. https://doi.org/10. 1016/j.cad.2006.08.004

3. Storga M, Andreasen MM, Marjanovic D (2008) The design ontology: foundation for the design knowledge exchange and management. J Eng Des 21(4):427-454. https://doi.org/10.1080/ 09544820802322557

4. Li Z, Zhou X, Wang WM, Huang G, Tian Z, Huang S (2018) An ontology-based product design framework for manufacturability verification and knowledge reuse. Int J Adv Manuf Technol 99:2121-2135. https://doi.org/10.1007/s00170-018-2099-2

5. Fenves S (2002) A Core Product Model for Representing Design Information. NIST Interagency/Internal Report (NISTIR), National Institute of Standards and Technology, Gaithersburg. https://doi.org/10.6028/NIST.IR.6736

6. Gruber TR (1993) A translation approach to portable ontology specifications. Knowl Acquis 5:199-220. https://doi.org/10.1006/ knac. 1993.1008

7. OWL 2 Web Ontology Language Document Overview (Second Edition). https://www.w3.org/TR/owl2-overview/. Accessed 02 Oct 2021

8. Bodenreider O (2008) Biomedical ontologies in action: role in knowledge management, data integration and decision support. Yearb Med Inform 17:67-79. https://doi.org/10.1055/s-00381638585

9. The Gene Ontology Consortium (2001) Creating the gene ontology resource: design and implementation. Genome Res 11(8):1425-1433. https://doi.org/10.1101/gr.180801

10. Kügler P, Marian M, Schleich B, Tremmel S, Wartzack S (2020) tribAIn: towards an explicit specification of shared tribological understanding. Appl Sci 10(13):4421. https://doi.org/10.3390/ app10134421

11. EMMO, EMMO: an Ontology for Applied Sciences. https:// emmc.info/emmo-info/. Accessed 02 Oct 2021

12. Pech F et al (2017) Semantic annotation of unstructured documents using concepts similarity. Sci Program. https://doi.org/10. $1155 / 2017 / 7831897$ 
13. Baader F et al (2017) An introduction to description logic. Cambridge University Press, Cambridge. https://doi.org/10.1017/ 9781139025355

14. Hahn O, Klemens U (1996) D 707-Fügen durch Umformen-Nieten und Durchsetzfügen-Innovative Verbindungsverfahren für die Praxis, Studiengesellschaft Stahlanwendung e.V

15. DVS-EFB 3420:2021-04, Clinching-basics

16. Meschut G, Janzen V, Olfermann $T$ (2014) Innovative and highly productive joining technologies for multi-material lightweight car body structures. J Mater Eng Perform 23(5):1515-1523

17. Wang M-h, G-q X, Li Z, Wang J-q (2017) Shape optimization methodology of clinching tools based on Bezier curve. Int $\mathrm{J}$ Adv Manuf Technol 24(1):2267-2280. https://doi.org/10.1007/ s00170-017-0987-5

18. Heimbs S, Nogueira AC, Hombergsmeier E, May M, Wolfrum J (2014) Failure behaviour of composite T-joints with novel metallic arrow-pin reinforcement. Compos Struct 110:16-28. https:// doi.org/10.1016/j.compstruct.2013.11.022

19. Feistauer EE, dos Santos JF, Amancio-Filho ST (2020) An investigation of the ultrasonic joining process parameters effect on the mechanical properties of metal-composite hybrid joints. Weld World 64:1481-1495. https://doi.org/10.1007/ s40194-020-00927-x

20. Plettke R et al (2014) A new process chain for joining sheet metal to fibre composite sheets. Key Eng Mater 611-612:1468-1475. https://doi.org/10.4028/www.scientific.net/kem.611-612.1468

21. Kraus M, Frey P, Kleffel T, Drummer D, Merklein M (2019) Mechanical joining without auxiliary element by cold formed pins for multi-material-systems. AIP Conference Proceedings 2113. https://doi.org/10.1063/1.5112570

22. Römisch D, Kraus M, Merklein M (2021) Experimental study on joining by forming of HCT590X $+\mathrm{Z}$ and EN-AW 6014 sheets using cold extruded pin structures. J Manuf Mater Process. https:// doi.org/10.3390/jmmp5010025

23. Graham DP, Rezai A, Baker D, Smith PA, Watts JF (2011) A hybrid joining scheme for high strength multi material joints. In: Proceeding of the 18th international conference on composite materials (ICCM18). ICC Jeju, Korea
24. Popp J, Kleffel T, Römisch D et al (2021) Fiber orientation mechanism of continuous fiber reinforced thermoplastics hybrid parts joined with metallic pins. Appl Compos Mater 28:951-972. https://doi.org/10.1007/s10443-021-09892-0

25. Ucsnik $S$ et al (2010) Experimental investigation of a novel hybrid metal-composite joining technology. Compos Part A Appl Sci Manuf 41(3):369-374. https://doi.org/10.1016/j.compositesa. 2009.11.003

26. Uschold M, Gruninger M (1996) Ontologies: principles, methods and applications. Knowl Eng Rev 11(2):93-136

27. DIN 8593-5:2003-09, Manufacturing processes joining-Part 5: Joining by forming processes; Classification, subdivision, terms and definitions. https://doi.org/10.31030/9500689

28. Feldmann K, Schöppner V, Spur G (2014) Handbuch Fügen, Handhaben. Carl Hanser Verlag, Montieren, Munich

29. SPARQL 1.1 Query Language, https://www.w3.org/TR/sparq111query/. Accessed 02 Nov 2021

30. Popp J, Kleffel T, Drummer D (2021) Influence of pin geometry on the joint strength of CFRT-metal hybrid parts with metallic pins. Join Plast 15(3-4):177-183

31. Hoekstra R (2010) The knowledge reengineering bottleneck. Semant Web 1(2):111-115

32. Kügler P, Marian M, Schleich B, Tremmel S, Wartzack S (2020) tribAIn: an ontology for knowledge representation in the domain of tribology. Proceedings of 61st. Tribologie-Fachtagung

Publisher's Note Springer Nature remains neutral with regard to jurisdictional claims in published maps and institutional affiliations. 